\title{
Deployment of Contact-Based Ultrasonic Thickness Measurements using Over-Actuated UAVs
}

\author{
Robert J. Watson ${ }^{1}$, S. Gareth Pierce ${ }^{1}$, Mina Kamel ${ }^{2}$, Dayi Zhang ${ }^{1}$, Charles N. Mac- \\ Leod $^{1}$, Gordon Dobie ${ }^{1}$, Gary Bolton ${ }^{3}$, Tariq Dawood ${ }^{4}$, \\ and Juan Nieto ${ }^{5}$ \\ ${ }^{1}$ Centre for Ultrasonic Engineering, University of Strathclyde, Glasgow, UK \\ \{robert.j.watson, s.g.pierce, dayi.zhang, charles.macleod, gordon.dobie\}@strath.ac.uk \\ ${ }^{2}$ Voliro AG, Zurich, Switzerland. mina.kamel@voliro.com \\ ${ }^{3}$ National Nuclear Laboratory ltd., Warrington, UK. gary.bolton@uknnl.com \\ ${ }^{4}$ EDF Energy R\&D UK Centre, Croydon, UK. tariq.dawood@edfenergy.com \\ ${ }^{5}$ Autonomous Systems Lab, ETH Zurich, Zurich, Switzerland. jnieto@ethz.ch
}

\begin{abstract}
Unmanned Aerial Vehicles (UAVs) are increasingly being utilized for the structural health assessment of on and off-shore structures. Visual inspection is the usual methodology for acquiring data from these structures, but there is often a need for contact based structural measurements, for example to assess local thickness on corroding structures. Conventional UAV platform dynamics have not traditionally allowed for such contact measurements. The limited dynamic control afforded by fixed plane rotor UAVs means that forward thrust (to apply contact forces for surface transduction) is only possible by tilting the whole platform, thus taking the UAV into a non-stationary state and limiting positional accuracy. An over-actuated UAV platform (with fully vectored thrust capability) may provide the required contact force for such thickness measurements whilst maintaining stable hovering next to the structure. The authors herein present a contact based ultrasonic thickness measurement technique, whereby an ultrasonic wheel probe deployed from a UAV was used to make single point and scanned measurements across a surface to provide a set of local thickness measurements. A $5 \mathrm{MHz}$, dry-coupled, dual-element, ultrasonic wheel probe is used to measure the thickness of an aluminum sample plate with thicknesses of $8.2 \mathrm{~mm}, 4.5 \mathrm{~mm}$ and $3.2 \mathrm{~mm}$, and a precision stepped calibration block with size from $31.5 \mathrm{~mm}$ to $17.5 \mathrm{~mm}$ in steps of $1 \mathrm{~mm}$, then steps of $0.1 \mathrm{~mm}$ down to $16.5 \mathrm{~mm}$ over a total length of $500 \mathrm{~mm}$. The thickness resolution obtainable from the ultrasonic wheel probe was typically $0.1 \mathrm{~mm}$, and the positional accuracy attained from the overactuated deployment platform was $16.6 \mathrm{~mm}$ when performing single point measurements.
\end{abstract}

Keywords: Novel Techniques, UAV Inspection, Ultrasound, Thickness Measurement, Automation, Remote Access 


\section{Introduction}

Structural Health Monitoring (SHM) is instrumental in the continued efficient operation and management of civil, aerospace, energy generation, and industrial infrastructure. It provides a quantitative assessment of functionality and residual useful life. Through active monitoring of asset attributes such as mechanical vibration, strain or displacement at periodic intervals, a profile of the initial "damage free" structure is created. Degradation through fatigue, or spontaneous damage, is then quickly detectable by statistical pattern recognition using current data. A current structure status, combined with accurate lifetime modelling, provides the opportunity to depart from timebased maintenance in favor of condition-based strategies. As such, repair activity can be scheduled when most cost effective. This can be during planned down-time, safely in advance of when usage measurement and residual life estimation predict failure.

Contemporary SHM takes many forms. The simplest techniques are based on visual inspection, but monitoring increasingly employs an array of permanently installed sensors distributed throughout the structure. Modal analysis techniques using arrays of accelerometers, strain sensors and global positioning systems detect and quantify changes to structural vibration modes brought on by damage [1]. Embracing the Internet of Things (IoT) paradigm, [2] details a scalable architecture for networking multiple lowcost, standardized transducers with the existing automated systems of an offshore platform. Use of a distributed sensor network has also been shown to detect changes to stimulated acoustic emissions indicative of crack formation in wind turbine blade edges [3]. Another example measured strain throughout wind turbine blades via embedded Fiber Bragg Grating (FBG) sensor system [4], detecting faults in the blades, tower and other components via their changes to their coupled resonances.

Common across multiple industrial sectors is corrosion monitoring. It is particularly prevalent in the oil and gas industry, seeing detailed ultrasonic corrosion mapping of pipelines and storage tanks [5]. Ultrasonic thickness measurement is similarly deployed in the nuclear industry to monitor of flow accelerated corrosion of pipes, with feasibility recently demonstrated for low power sensors mounted under insulation that are individually polled by an inductive wand [6].

However, it is not always practical to embed a full suite of SHM sensors within a structure. Instead, periodic inspection may be conducted in person or with mobile robotic assistance. Surface crawler and UAV solutions are widely adopted to gather repeatable measurements in locales with time consuming or otherwise hazardous physical entry requirements [7].

SHM applications supported by UAV typically take the form of remote visual inspection. A pertinent example is found in the case of wind turbines, where UAV captured images are a primary means of assessing the state of the composite blade material, prone to weather based leading edge erosion and structural fatigue due to cyclical loading [8], [9]. As edge erosion is a surface exposed damage mode, UAVs are readily able to detect this and other similar modes visually, screening turbines for significant degradation requiring immediate remediation and precluding some instances of manned access. More advanced applications address the loss of context in discrete images via photogrammetric reconstruction [10], producing a 3D model and tracking geometry 
changes through periodic inspection. Similar processes have been deployed for the inspection of bridges [11], [12], topographic surveys [13], nuclear storage cannisters [14] and industrial chimneys [15].

However, when assessing internal structural status, visual inspection is limited to indirect qualitative inference using features like staining or surface breaking cracks. To provide subsurface health status, most SHM methods require physical contact with the structure. This has previously presented a problem to aerial assessment methods. Aerodynamic features such as near surface turbulence and vortices downwind of tall structures require highly dynamic corrective action by a UAV agent. Challenges are exacerbated by the need to maneuver the multirotor to reject such disturbance while simultaneously applying force sufficient to hold a sensor in stationary contact as readings are taken. Combined with the variations in orientation for access around the complex surface geometries of civil and industrial structures, aerial interaction for SHM is a nontrivial challenge.

Addressing such, presented herein is a multirotor UAV capable of conducting remote, aerial, ultrasonic thickness measurement with a dry-coupling wheel probe. This may be deployed in sequential discrete point measurement and linear scanning modalities. Demonstrations are made of this capability, supported by quantitative assessment of the impact of the floating platform on the ultrasonic thickness measurement accuracy and the strategy used to localize these measurements relative to the target.

The remainder of this paper is structured as follows: Section 2 examines the methodology for remote ultrasonic SHM by an aerial multirotor agent, and Section 3 details the results of laboratory flight testing using this system. Finally, potential future work and conclusions drawn from these findings are outlined in Section 4 and 5, respectively.

\section{Inspection System Overview}

\subsection{Voliro Multirotor UAV}

The Voliro aerial manipulation platform [16], [17] of Fig. 1 grants a unique capability to address the challenges of aerial SHM. As an over-actuated system, it has more actuators than dimensions of its aerial pose state. These effectors take the form of two main propeller pairs on arms extending from the craft center of mass and a reversible thrust tail rotor. As indicated, both propeller pairs may be independently oriented in two rotational degrees of freedom (DoF) during flight. This allows arbitrary wrench generation and force and torque control across all translational and rotational axes, permitting stable flight at non-horizontal attitudes. The Voliro thus benefits from an enhanced dynamic freedom over conventional multirotor platforms with unidirectional thrust which must re-orient the entire craft to change their net thrust vector. Additionally, the Voliro may maintain body orientation while altering its thrust to exert a maximum of $3 \mathrm{~kg}$ in all directions. This permits efficient rejection of near-surface aerodynamic disturbances during stationary surface contact.

As proximity to large structures can deny signals from Global Navigation Satellite Systems (GNSS) via radio wave blocking and introduce multi-path errors, the Voliro may 
operate with several positioning technologies. Within the indoor laboratory environment translation and rotational 6 DoF pose estimation is provided by a Vicon motion tracker system [18]. This may be purposefully degraded to have accuracy similar to a typical GNSS system, allowing representative performance during testing. In flight the world frame positioning data is supplied to the UAV controller over Wi-Fi via a ground station PC linked to an onboard intel 17 NUC, both running the Robotic Operating System (ROS). Pose data (and other telemetry) is then shared with a Pixhawk Flight controller via a bi-directional serial link. The Pixhawk fuses pose data with measurements from the onboard Inertia Measurement Unit (IMU) to derive an accurate position estimate, as with GNSS data. It also regulates UAV position and orientation in accordance with pilot input. Power is supplied to the UAV from an onboard battery, but for convenience and extended flight duration, may alternately be provided from a domestic electrical outlet via a high-voltage DC tether.

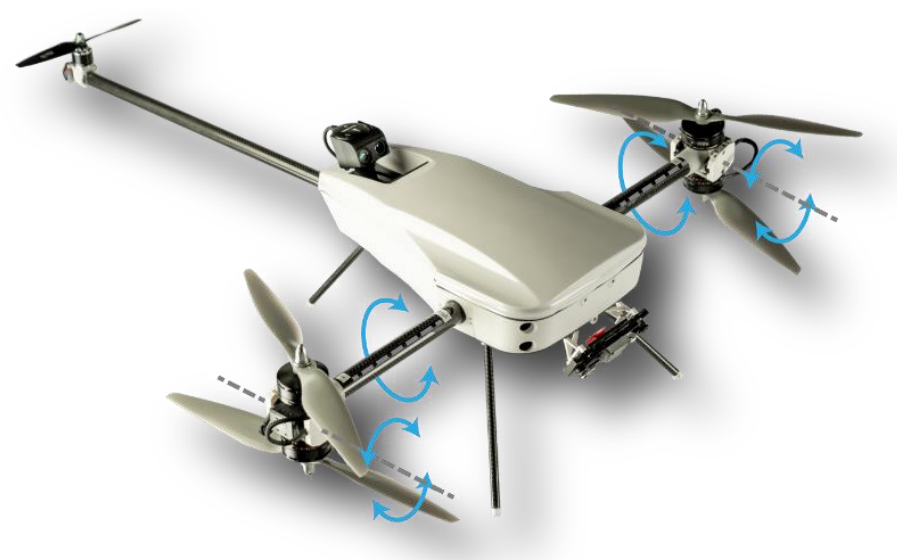

Fig. 1. The over-actuated Voliro multirotor UAV. Annotations show the additional degrees of freedom about which the main propeller pairs may be independently rotated.

\subsection{Ultrasonic Instrumentation}

Lightweight ultrasonic instrumentation was embedded aboard the UAV in support of contact based SHM per Fig. 2. This proprietary ultrasonic pulse generator and receiver was developed for integration within small form factor mobile robots [19] and drove a $25 \mathrm{~mm}$ diameter, dry-coupling, dual-element, $5 \mathrm{MHz}$ wheel probe [20]. The dedicated transmit and receive, dual-element configuration is recommended for corrosive measurement per international standards [21], [22], reducing sensitivity to surface roughness and dead-zone issues in thin samples. Further, the acoustic impedance and low attenuation of the dry-coupling rubberized tire enabled ultrasonic coupling without an onboard reservoir of the conventional liquid gel. This reduced instrumentation payload and precluded issues of changing center of mass as the gel was dispensed. A total mass of $132 \mathrm{~g}$ and bounding dimensions of $136 \mathrm{~mm}$ x $61 \mathrm{~mm}$ x $33 \mathrm{~mm}$ made this combination well suited to UAV platform constraints. 


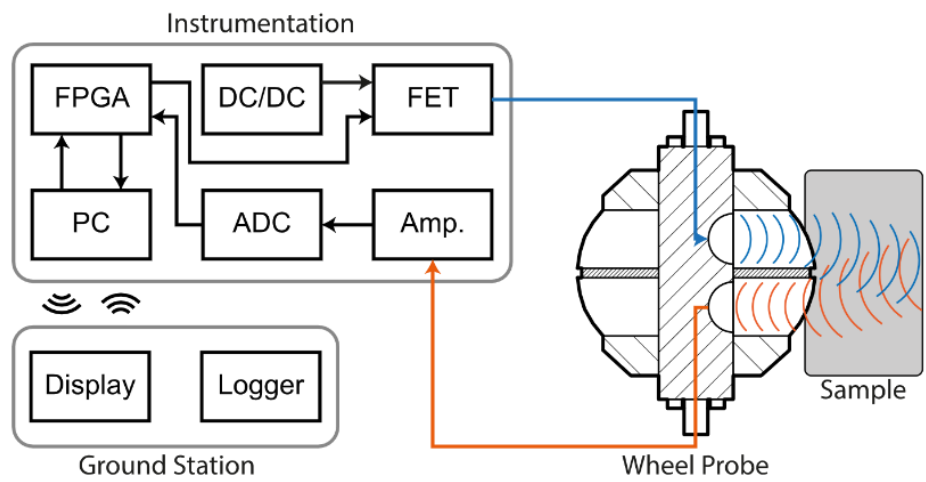

Fig. 2. Ultrasonic inspection system diagram. A cross-sectional view of the wheel probe shows the propagation route of the electrical and ultrasonic measurement signals through its dual piezoelectric elements and the sample under test.

Ultrasonic pulse transmission was initiated when the Field Programmable Gate Array (FPGA) received a command from the onboard PC. This caused the Field Effect Transistor (FET) to deliver a $180 \mathrm{~V} \mathrm{DC/DC} \mathrm{boost} \mathrm{converted} \mathrm{single} \mathrm{pulse} \mathrm{excitation} \mathrm{to} \mathrm{one}$ of the piezo elements within the wheel probe axel, and generating ultrasonic wave packets with $5 \mathrm{MHz}$ center frequency. In the receiving pathway, ultrasonic signals reflected by the sample were converted to electrical pulses by the second piezo element. After passing through a variable gain transimpedance amplifier, the full A-scan waveform was captured by an 8-bit Analog to Digital Converter (ADC), operating at 100 Msps. This was stored in a 8192 byte buffer in FPGA memory, granting a maximum signal length of just under $82 \mu$ s duration at a variable delay following transmission. A-scans were transferred to the onboard PC via USB 2 connection and conditioned by a $60 \mathrm{~dB}$ band-pass filter attenuating noise outside $\pm 2 \mathrm{MHz}$ of the transducer center frequency.

There, a custom interface node distributed them to the UAV ROS network. This node enabled logging and real-time inspection signal visualization from the ground station. It also remotely triggered the transceiver at a $100 \mathrm{~Hz}$ pulse repetition frequency. Other ROS modules aided aggregation of measurements with UAV pose data, encoding their position relative to the target structure to enhance inspection context.

\subsection{Experimental Setup}

Prior to adoption, any novel SHM approach must be quantitatively profiled for accuracy. Thus, the aerial ultrasonic inspection system has been assessed within a controlled laboratory environment, wherein aspects of performance can be independently tested. Empirical trials are conducted against the rolled aluminum samples of Fig. 3, having known geometry and containing features representative of corrosive thickness loss.

The flat plate sample had two pockets milled from its rear surface representative of large scale corrosion. It was used to test positioning repeatability and measurement performance in thin samples approaching the accepted practical minimum thickness limit of one wavelength [21] (under $1.3 \mathrm{~mm}$ in aluminum). The bar sample was precision 
machined to give a thickness staircase decreasing from $31.5 \mathrm{~mm}$ in $1 \mathrm{~mm}$ steps until a thickness of $17.5 \mathrm{~mm}$, then in $100 \mu \mathrm{m}$ steps to $16.5 \mathrm{~mm}$. It was used to test the ability of the system to perform linear scans. Owing to manufacturing tolerances, the dimensions of both samples vary slightly from the nominal dimensions of Fig. 3. Consequently, the "as built" dimensions, determined by micron caliper measurements taken at regular intervals across the samples, were used as the reference for all considerations of ultrasonic thickness measurement accuracy. To assess the effects capability on the inspection process of non-horizontal, over-actuated flights, the samples were mounted vertically or on the underside of a $45^{\circ}$ overhang. This served as an analog to an inspection scenario amid complex industrial structures.

(a)

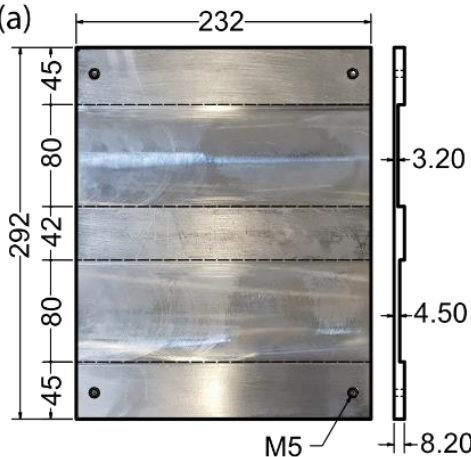

(b)

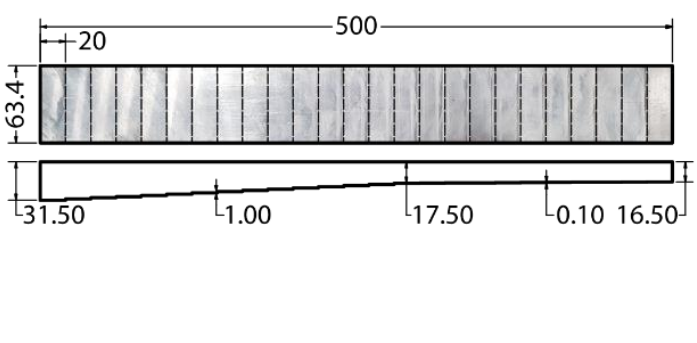

Fig. 3. Photographs of the rear surface of the aluminum plate (a) and bar (b) samples. Annotations give nominal geometry and cross-section profile. All dimensions are in millimeters.

\section{Results}

\subsection{Multidirectional Structure Inspection}

To obtain ultrasonic measurements with the dry-coupling probe, the UAV must apply pressure to deform the rubber tire and expunge the air layer at its sample interface. By experimentation, this tire material and surface roughness combination required a minimum of around $20 \mathrm{~N}$. Further, the probe orientation must be within $10^{\circ}$ of surface normal to align its piezo elements with the acoustically coupled region of the tire.

As depicted in Fig. 4a and b, the over-actuated UAV was successfully re-orientated about its pitch axis to match the inspection surface normal by actuation of the propeller arms in mid-flight. It then generated additional force into the surface to meet the coupling criteria and capture the ultrasonic A-scans of Fig. $4 \mathrm{c}$ and $\mathrm{d}$ while in stable contact, here sustained for over $2.5 \mathrm{~s}$. The A-scan amplitudes varied with minor changes in coupling, but have sufficient signal to noise ratio (SNR) for thickness measurement. 
(a)

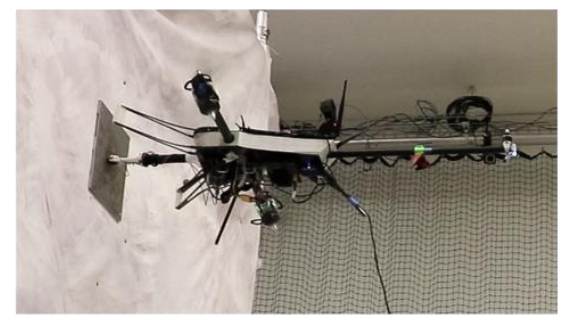

(c)

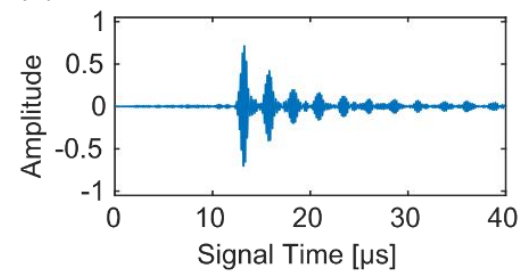

(b)

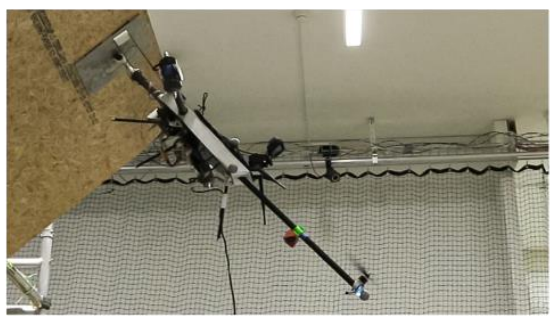

(d)

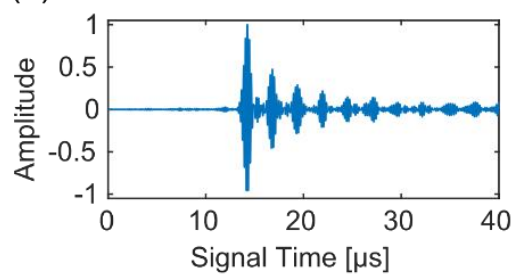

Fig. 4. Inspections are performed against an aluminum test piece in (a) vertical and (b) overhanging orientations. Normalized ultrasonic A-scan signals captured at the center of the sample in each of these poses are given in (c) and (d) respectively.

\subsection{Ultrasonic Inspection Accuracy}

Thickness, $d$, may be derived from an ultrasonic A-scan by Eq. (1).

$$
d=v t / n
$$

Where: $v$ is the material speed of sound, $t$ is the signal time of flight, and $n$ is the number of transits made across the thickness of interest. The time of flight between subsequent back-wall echoes corresponds to two transits of the sample thickness and is computed via auto-correlation. This yields a symmetrical waveform immune to changes in the first-echo position caused by variation in tire deformation force. Applying the Hilbert Transform then demodulates the signal, removing the $5 \mathrm{MHz}$ piezo center frequency and yielding an amplitude envelope. The time of flight corresponds to the time of the first local maximum with positive lag, found by simple peak detection algorithm.

The speed of sound for each sample completes Eq. (1) and was evaluated by recording the time of flight across a known thickness. From the average of 2000 readings of the plate and bar samples, the measured speeds of sound were $6417 \mathrm{~m} / \mathrm{s}$ and $6405 \mathrm{~m} / \mathrm{s}$, respectively, agreeing with the standard range for rolled aluminum [23].

Using the recorded ultrasound data from repeated contact inspections across multiple flight trials, system accuracy is quantified by the error between the numerical thickness and the "as built" sample reference geometry. The measurement error distribution from the vertical and overhanging plate sample inspections is described in Table 1. 
Table 1. Error distribution statistics in ultrasonic thickness versus reference geometry.

\begin{tabular}{lccccc}
\hline $\begin{array}{l}\text { Sample } \\
\text { Orientation }\end{array}$ & $\begin{array}{c}\text { Number of } \\
\text { Samples }\end{array}$ & $\begin{array}{c}\text { Mean Error } \\
{[\mathrm{mm}]}\end{array}$ & $\begin{array}{c}\text { Mean Absolute } \\
\text { Error } \\
{[\mathrm{mm}]}\end{array}$ & $\begin{array}{c}5^{\text {th }} \text { Percentile } \\
\text { Error }[\mathrm{mm}]\end{array}$ & $\begin{array}{c}9^{\text {th }} \text { Percentile } \\
\text { Error }[\mathrm{mm}]\end{array}$ \\
\hline Vertical & 3868 & -0.048 & 0.099 & -0.218 & 0.102 \\
\hline $45^{\circ}$ Overhang & 1482 & -0.054 & 0.092 & -0.225 & 0.160 \\
\hline
\end{tabular}

The error metrics for both orientations were broadly similar, indicating performance was maintained across interaction orientations, though $5^{\text {th }}$ to $95^{\text {th }}$ percentile range indicates a slight increase in variability in the overhanging case. With the mean absolute error close to $0.1 \mathrm{~mm}$, the single ultrasonic measurement accuracy was comparable to typical hand-held, commercial thickness gauges [24], [25]. Further improvement is attainable via averaging of multiple readings. In both trials, mean error approached the theoretical thickness resolution limit of $0.032 \mathrm{~mm}$ given by the instrumentation sampling rate and speed of sound in aluminum. Accuracy was within the $\pm 0.5 \mathrm{~mm}$ reported for typical in situ manual thickness gauging [26], [27].

\subsection{Positioning Encoding Uncertainty}

For this assessment, a total of 11 measurement interactions were completed with the vertical plate sample, each holding stationary contact for at least $2 \mathrm{~s}$. Null ultrasonic readings acquired out of contact with the surface were discarded by evaluation of their maximum amplitude relative to a noise threshold. The position of the remaining signals on the sample were then derived by projection from the UAV pose at the time of measurement, using the direction of the probe arm, to the point of intersection with the sample face. The uncertainty associated with the position encoding of these measurements when considering the UAV internal pose estimate was then evaluated by considering their distribution. As the probe position was constant during contact, taking the linear displacement of each ultrasonic reading from the mean of those in the same interaction allowed comparison across multiple contact instances for a total of 3874 samples. The resultant distribution given in Table 2 provided a measure for confidence in the position of any single reading within the context of the SHM process.

Table 2. Position uncertainty distribution statistics.

\begin{tabular}{lcccc}
\hline $\begin{array}{l}\text { Number of } \\
\text { Samples }\end{array}$ & $\begin{array}{r}\text { Mean } \\
{[\mathrm{mm}]}\end{array}$ & $\begin{array}{c}90^{\text {th }} \text { Percentile } \\
{[\mathrm{mm}]}\end{array}$ & $\begin{array}{c}95^{\text {th }} \text { Percentile } \\
{[\mathrm{mm}]}\end{array}$ & $\begin{array}{c}\text { Maximum } \\
{[\mathrm{mm}]}\end{array}$ \\
\hline 3874 & 5.13 & 9.49 & 11.01 & 16.55 \\
\hline
\end{tabular}

From the worst case position encoding deviation of $16.55 \mathrm{~mm}$ recorded for any one measurement, it may be concluded that the internal position estimate of the system is sufficient for localization of large-scale, flat pitting, uniform, or mesa-type corrosive 
thickness loss features to within an acceptable margin. However, if the application entails detailed corrosion mapping of features at a scale much below $10 \mathrm{~mm}$, then additional measurements should be taken to increase positional certainty.

\subsection{Scanning Acquisition}

Leveraging the Voliro capability for dynamic environmental interactions, scanning measurements were conducted. Rolling the wheel probe in continuous contact along the surface of the stepped bar sample, and combining the recorded A-scans, the crosssectional B-scan view depicted in figure 5 was produced.

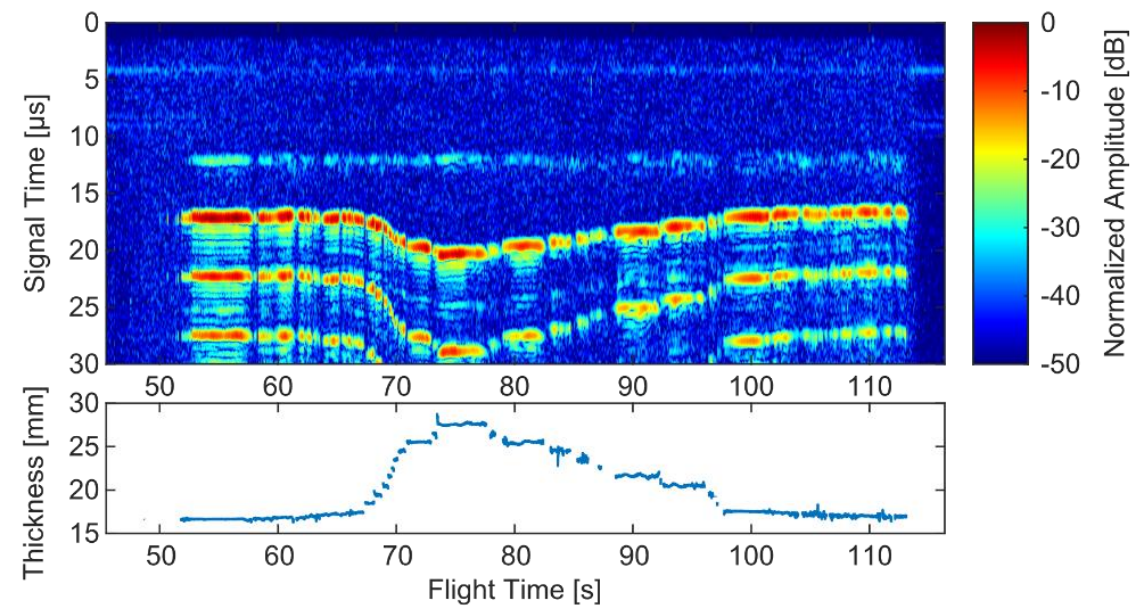

Fig.5. A B-scan cross-sectional view captured by rolling the wheel probe along the stepped bar sample surface. This shows variation in sample thickness with time along the UAV flightpath over two passes across the sample, from the thinnest end, to the thickest and back again in under $65 \mathrm{~s}$. Derived numerical thickness is also plotted.

Within figure 5, the relative thickness of the part under inspection is clearly visible in the time difference between the front-wall echo (received after around $12 \mu \mathrm{s}$, with slight variation due to changes in tire compression) and the first back-wall echo. It is also observed in the time between subsequent backwall echoes. All thickness features within the swept path along the bar sample were successfully captured in multiple data points, however the scale of the $0.1 \mathrm{~mm}$ steps relative to the ultrasonic measurement accuracy means they appear more as a linear gradient. Asymmetry in the B-scan image arises owing to variation in the UAV rolling speed between the outward and return motions. This was a due to a sudden reduction in rolling resistance at the transition from the static to dynamic mode. The resultant burst of motion was challenging to sustain within the acoustic coupling criteria. In spite of this the integrated system maintained the acoustic coupling conditions with sufficient consistency to resolve thickness features at the surface parallel pitch under test over two passes. 


\section{$4 \quad$ Future Work}

In light of these findings, possible avenues for future work are identified. Development of the scanning process to improve the speed and stability of surface traversal would permit the time efficient acquisition of densely sampled corrosion maps. Additional surface-relative position sensors could improve position encoding precision, augmenting resolution of small features and repeatability between scans. Additional possibilities may be found in the incorporation of sensing modalities beyond the ultrasonic testing used here. Such developments increase applicability to SHM scenarios where time intensive and hazardous inspection methods would conventionally be deployed.

\section{Conclusions}

In summary, a prototype for an aerial contact based inspection system to support remote structural monitoring has been presented. Challenges of stability and repeatability within the context of contact-based ultrasonic inspection from aboard a UAV have been addressed. Employing an over-actuated aerial manipulator platform in conjunction with a dry-coupling ultrasonic wheel probe, sustained interaction supportive of remote ultrasonic thickness measurement has been demonstrated in multiple flight orientations, successfully interacting with vertical, inclined, and overhanging structural features. These scenarios are supportive of corrosion monitoring activity in a range of SHM applications. Further, this article has presented a quantitative characterization of aerial ultrasonic thickness measurement accuracy and the ability of the system to locate these data relative to the inspection target. Lastly, the ability of the integrated UAV system to perform rolling, time-encoded, cross-sectional B-scan profiling has been documented and suggestions made for further development in future work.

\section{Acknowledgements}

This work was supported by EPSRC funding under the iCASE program, Ref: EP/R512114/1, in conjunction with EDF Energy and the National Nuclear Laboratory.

\section{References}

[1] A. Deraemaeker, "Vibration Based Structural Health Monitoring Using Large Sensor Arrays: Overview of Instrumentation and Feature Extraction Based on Modal Filters," in New Trends in Vibration Based Structural Health Monitoring, A. Deraemaeker and K. Worden, Eds. Vienna: Springer, 2010, pp. 19-54.

[2] M. Sayed, M. Nemitz, S. Aracri, A. McConnell, R. McKenzie, and A. Stokes, "The Limpet: A ROS-Enabled Multi-Sensing Platform for the ORCA Hub," Sensors, vol. 18, no. 10, p. 3487, Oct. 2018, doi: 10.3390/s18103487. 
[3] C. Beale, C. Niezrecki, and M. Inalpolat, "An adaptive wavelet packet denoising algorithm for enhanced active acoustic damage detection from wind turbine blades," Mech. Syst. Signal Process., vol. 142, p. 106754, Aug. 2020, doi: 10.1016/j.ymssp.2020.106754.

[4] T. J. Arsenault, A. Achuthan, P. Marzocca, C. Grappasonni, and G. Coppotelli, "Development of a FBG based distributed strain sensor system for wind turbine structural health monitoring," Smart Mater. Struct., vol. 22, no. 7, p. 075027, Jun. 2013, doi: 10.1088/09641726/22/7/075027.

[5] C. F. Britton, "4.36 Corrosion Monitoring and Inspection," in Shreir's Corrosion, vol. 4, Bob Cottis, Michael Graham, Robert Lindsay, Stuart Lyon, Tony Richardson, David Scantlebury, and Howard Stott, Eds. Elsevier, 2010, p. 50.

[6] A. Tamura et al., "A non-contact ultrasonic sensor for pipe-wall thinning inspection of nuclear power plants," in 45th Annual Review of Progress in Quantitative Nondestructive Evaluation, Portland, OR, USA, May 2019, vol. 2102, p. 6, doi: 10.1063/1.5099799.

[7] D. Lattanzi and G. Miller, "Review of Robotic Infrastructure Inspection Systems," J. Infrastruct. Syst., vol. 23, no. 3, p. 04017004, Sep. 2017, doi: 10.1061/(ASCE)IS.1943555X.0000353.

[8] G. Morgenthal and N. Hallermann, "Quality Assessment of Unmanned Aerial Vehicle (UAV) Based Visual Inspection of Structures,” Adv. Struct. Eng., vol. 17, no. 3, pp. 289302, Mar. 2014, doi: 10.1260/1369-4332.17.3.289.

[9] L. Wang and Z. Zhang, "Automatic Detection of Wind Turbine Blade Surface Cracks Based on UAV-Taken Images,” IEEE Trans. Ind. Electron., vol. 64, no. 9, pp. 7293-7303, Sep. 2017, doi: 10.1109/TIE.2017.2682037.

[10] D. Zhang, R. Watson, G. Dobie, C. MacLeod, A. Khan, and G. Pierce, "Quantifying impacts on remote photogrammetric inspection using unmanned aerial vehicles," Eng. Struct., vol. 209, p. 109940, Apr. 2020, doi: 10.1016/j.engstruct.2019.109940.

[11] J. Seo, L. Duque, and J. Wacker, "Drone-enabled bridge inspection methodology and application," Autom. Constr., vol. 94, pp. 112-126, Oct. 2018, doi: 10.1016/j.autcon.2018.06.006.

[12] A. Khaloo, D. Lattanzi, K. Cunningham, R. Dell'Andrea, and M. Riley, "Unmanned aerial vehicle inspection of the Placer River Trail Bridge through image-based 3D modelling," Struct. Infrastruct. Eng., vol. 14, no. 1, pp. 124-136, Jan. 2018, doi: 10.1080/15732479.2017.1330891.

[13] M. R. James, S. Robson, S. d'Oleire-Oltmanns, and U. Niethammer, “Optimising UAV topographic surveys processed with structure-from-motion: Ground control quality, quantity and bundle adjustment," Geomorphology, vol. 280, pp. 51-66, Mar. 2017, doi: 10.1016/j.geomorph.2016.11.021.

[14] R. A. Clark et al., "Autonomous and scalable control for remote inspection with multiple aerial vehicles," Robot. Auton. Syst., vol. 87, pp. 258-268, Jan. 2017, doi: 10.1016/j.robot.2016.10.012.

[15] J. Quenzel, M. Nieuwenhuisen, D. Droeschel, M. Beul, S. Houben, and S. Behnke, “Autonomous MAV-based Indoor Chimney Inspection with 3D Laser Localization and Textured Surface Reconstruction,” J. Intell. Robot. Syst., May 2018, doi: 10.1007/s10846-018-0791y. 
[16] M. Kamel et al., "The Voliro Omniorientational Hexacopter: An Agile and Maneuverable Tiltable-Rotor Aerial Vehicle," IEEE Robot. Autom. Mag., vol. 25, no. 4, pp. 34-44, Dec. 2018, doi: 10.1109/MRA.2018.2866758.

[17] "Voliro Airborne Robotics." https://www.voliro.com/ (accessed Jun. 29, 2020)

[18] Vicon, "Tracker Motion Capture Software for VR and Object Tracking." http://www.vicon.com/products/software/tracker (accessed Jun. 30, 2020).

[19] C. N. Macleod, G. Dobie, S. G. Pierce, R. Summan, and M. Morozov, "Machining-Based Coverage Path Planning for Automated Structural Inspection," IEEE Trans. Autom. Sci. Eng., vol. 15, no. 1, pp. 202-213, Jan. 2018, doi: 10.1109/TASE.2016.2601880.

[20] "Home | Eddyfi." https://www.eddyfi.com (accessed Oct. 31, 2019).

[21] Technical Committee ISO/TC 135, "Non-destructive testing - Ultrasonic thickness measurement (ISO 16809:2017)," International Standards Organisation, Geneva, Switzerland, Nov. 2017. Accessed: Nov. 01, 2019. [Online]. Available: https://www.iso.org/stand$\operatorname{ard} / 72430 . h t m l$.

[22] E07 Committee, "E797: Standard Practice for Measuring Thickness by Manual Ultrasonic Pulse-Echo Contact Method," ASTM International. doi: 10.1520/E0797_E0797M-15.

[23] Dwight E. Gray, American Institue of Physics Handbook, 3rd ed. New York, NY, USA: McGraw-Hill, 1972.

[24] "27MG Ultrasonic Thickness Gage." https://www.olympusims.com/en/27mg/\#!cms[tab]=\%2F27mg\%2Fspecifications (accessed Jun. 29, 2020).

[25] "Ultrasonic Thickness Tester PCE-TG 50 | PCE Instruments." https://www.pce-instruments.com/english/measuring-instruments/test-meters/ultrasonic-tester-ultrasonic-testingpce-instruments-ultrasonic-thickness-tester-pce-tg-50-det_5233277.htm (accessed Jun. 29, 2020).

[26] J. C. Drury, "Corrosion Monitoring and Thickness Measurement - What are we doing wrong?," Insight - Non-Destr. Test. Cond. Monit., vol. 39, no. 1, pp. 17-20, 1997.

[27] D. R. Weier and A. F. Pardini, "Evaluation of UT Wall Thickness Measurements and Measurement Methodology," Pacific Northwest National Laboratory, Richland, Washington 99352, US, PNNL-16828 Rev. 0, 1035013, Oct. 2007. doi: 10.2172/1035013. 